\title{
Comparison of diagnostic accuracy of transvaginal ultrasound with laparoscopy in the management of patients with adnexal masses
}

\author{
Theodoros D. Theodoridis • Leonidas Zepiridis • \\ Themistoklis Mikos · Grigoris F. Grimbizis · \\ Konstantinos Dinas • Apostolos Athanasiadis · John N. Bontis
}

Received: 7 October 2008 / Accepted: 9 February 2009 / Published online: 5 March 2009

(c) The Author(s) 2009. This article is published with open access at Springerlink.com

\begin{abstract}
Purpose The aims of this study was (a) to compare the diagnostic accuracy of ultrasound examination with laparoscopic findings and both with the gold standard (histology) in the management of benign ovarian lesions, and (b) to assess the feasibility of laparoscopy in their diagnosis and management.

Methods Prospective, comparative study (Canadian Task Force Classification II-2). A total of 117 women 1559 years old were examined at outpatient department and had transvaginal ultrasound assessment. Ninety-eight women (three postmenopausal) with 105 cystic ovarian lesions met inclusion criteria and underwent operative laparoscopy. Histology was performed in all cases.

Results Although laparoscopy showed an overall higher performance compared to transvaginal ultrasound, statistically significant difference was found only in the detection of endometriomas compared to ultrasound $(P=0.004$ for sensitivity and $P=0.046$ for specificity).

Conclusion Laparoscopy exhibits higher diagnostic accuracy, especially in endometriomas, compared to transvaginal
\end{abstract}

T. D. Theodoridis · L. Zepiridis · T. Mikos · G. F. Grimbizis ·

K. Dinas · A. Athanasiadis - J. N. Bontis

1st Department of Obstetrics and Gynecology,

Aristotle University of Thessaloniki,

Thessaloniki, Greece

T. D. Theodoridis · L. Zepiridis · T. Mikos · G. F. Grimbizis ·

J. N. Bontis

Endoscopy Unit, General Clinic, Thessaloniki, Greece

T. D. Theodoridis $(\square)$

8, Agias Theodoras Street, 54623 Thessaloniki, Greece

e-mail: theodtheo@yahoo.gr scan. Laparoscopic diagnosis appears to be safe and accurate. Conservative laparoscopic management of benign adnexal masses is safe and with low morbidity.

Keywords Laparoscopy · Adnexal masses ·

Ovarian cysts · Transvaginal scan · Endometriomas

\section{Introduction}

The application of imaging techniques in routine gynecological practice has lead to an increased detection rate of adnexal masses. However, accurate diagnosis is commonly obscured by the complexity of clinical and imaging features of these lesions. Clinician's primary goal is to rule out malignancy, as this demands immediate management in order to prevent devastating results. The risk of an ovarian tumor being malignant is estimated to be $7-13 \%$ in premenopausal and $8-45 \%$ in postmenopausal women [1]. The risk of ovarian malignancy in women undergoing laparoscopy for preoperatively benign appearing ovarian tumors ranges from 0.1 to $4.2 \%$ and increases in elder patients [2-5].

Ultrasound scan has been widely used for ovarian pathology screening. A number of scoring systems has been proposed in order to facilitate early detection of malignant ovarian lesions [6-9]. Sonographic scoring of the ovarian lesion appears to have high sensitivity (89-100\%) and specificity (73-83\%), moderate positive predictive value $(37-46 \%)$ and excellent negative predictive value (96$100 \%)[6,7]$.

Nevertheless, it seems that the most accurate way to detect ovarian malignancies is the combination of laparoscopic inspection of the peritoneal cavity with intraoperative biopsy [2]. The laparoscopic management of ovarian 
masses is currently one of the most intriguing issues of contemporary gynecology. Until recently, the optimum approach for the management of ovarian tumors was laparotomy. The increasing experience in the use of laparoscopy during the last 15 years has altered the way of treating adnexal tumors in favor of minimally invasive techniques. Advantages of laparoscopy compared to laparotomy include shorter hospital stay, smaller percentage of postoperative adhesions, less impact on quality of life, and the overall decreased financial burden $[10,11]$. Moreover, current trends indicate that ovarian lesions in young women, with or without subfertility, warrant mainly conservative management, and if operation cannot be avoided, then application of minimal invasive surgery is mandatory.

The aim of this study was to compare the diagnostic accuracy of ultrasound examination with laparoscopic findings and both with the gold standard (histology) in the management of benign ovarian lesions, and to assess the feasibility of laparoscopy in their diagnosis and management.

\section{Materials and methods}

Ethical approval for this prospective, comparative study was obtained by the local ethical committee as appropriately.

A total of 117 women 15-59 years old were examined in the outpatient department of a tertiary centre (1st Department of Obstetrics and Gynecology, Aristotle University of Thessaloniki, Greece) and in an affiliated private hospital (General Clinic, Thessaloniki, Greece) from September 2001 to September 2005.

Presenting symptoms, anamnesis, clinical bimanual examination, and transvaginal ultrasound scan (TVS), were taken during the first visit in the outpatient clinic. The commonest presenting symptoms were pelvic pain, menstrual disorders, dysmenorrhoea, dyspareunia, subfertility, and routine check-up in asymptomatic women.

Inclusion criteria for this study were the presence of sonographically diagnosed cystic ovarian mass in both premenopausal and postmenopausal women. Detailed ultrasound examination (Ultramark 4 Plus Advanced Technology Laboratories, Bothell, WA, USA, and Logic 200 Q, General Electric, vaginal probe with $5 \mathrm{MHz}$ frequency) was performed by experienced sonographers and Sassone score was calculated for each subject. Postmenopausal women with Sassone score $>9$ were excluded from the study [6].

All subjects were rescanned 2 months after the initial visit in order to identify functional ovarian lesions. Nineteen women 19-41 years old were excluded from the study protocol as subsequent ultrasound examination did not confirm the presence of the originally identified mass (functional ovarian cysts).

Ninety-eight women (three postmenopausal) with 105 cystic ovarian lesions were finally recruited for further laparoscopic management. Body mass index ranged from 17 to $35 \mathrm{~kg} / \mathrm{m}^{2}$. Diameter of the ovarian masses ranged from 2.9 to $13.5 \mathrm{~cm}$ (Table 1$)$.

All 98 women underwent operative $\mathrm{CO}_{2}$ laparoscopy. Pneumoperitoneum was established with the Veress needle, except two cases with previous midline laparotomy that pneumoperitoneum was set with open laparoscopy. After trocar insertion, peritoneal fluid cytology was obtained routinely. Laparoscopic cystectomy was performed as previously described [5]. The cyst was removed by means of an endobag and was histologically examined. Suction irrigation of the peritoneal cavity and secure hemostasis concluded each laparoscopic operation.

Statistics were performed with the use of SPSS version 11.0 and Epi info for Windows Database and Statistics for Public Health Professionals [Centers for Disease Control and Prevention (CDC)]. Student's $t$-test and Fisher's exact

Table 1 Patients' age and BMI, sonographic diameter of the masses, classified according to the histological diagnosis

\begin{tabular}{lrlll}
\hline & $n$ & Age (years) $($ mean $\pm \mathrm{SD})$ & BMI $\left(\mathrm{kg} / \mathrm{m}^{2}\right)(\mathrm{mean} \pm \mathrm{SD})$ & Diameter $(\mathrm{cm})(\mathrm{mean} \pm \mathrm{SD})$ \\
\hline Simple cyst & 32 & $32.4 \pm 19.6$ & $22 \pm 7.0$ & $5.07 \pm 2.92$ \\
Paraovarian cyst & 4 & $23.5 \pm 16.6$ & $27.7 \pm 5.2$ & $7.5 \pm 8.16$ \\
Endometrioma & 37 & $30.8 \pm 14.6$ & $23.3 \pm 8.8$ & $5.11 \pm 3.28$ \\
Serous cystadenoma & 8 & $32.4 \pm 26.0$ & $25.7 \pm 9.6$ & $6.04 \pm 2.78$ \\
Mucinous cystadenoma & 4 & $42.4 \pm 26.0$ & $27 \pm 8.6$ & $5.12 \pm 2.5$ \\
Dermoid cyst & 15 & $26.3 \pm 7.2$ & $22.4 \pm 7.0$ & $5.15 \pm 2.24$ \\
Cystic adenofibroma & 1 & 37 & 30 & 5.0 \\
Corpus luteum cyst & 2 & $31.5 \pm 15.6$ & $20.5 \pm 1.4$ & $5.75 \pm 0.7$ \\
Borderline tumor & 2 & $27.5 \pm 3.0$ & $22 \pm 2.8$ & $6 \pm 1.4$ \\
Total & 105 & $31.04 \pm 18.48$ & $23.09 \pm 8.32$ & $5.29 \pm 3.26$ \\
\hline
\end{tabular}

$n$ Number of cysts, $B M I$ body mass index, $S D$ standard deviation 
Table 2 Presenting symptoms of the patients included in the study

\begin{tabular}{|c|c|c|c|c|c|c|}
\hline & Pelvic pain & Menstrual disorders & Dysmenorrhoea & Dyspareunia & Subfertility & Annual visit \\
\hline Simple cyst & 18 & 8 & 1 & 2 & 1 & 15 \\
\hline Paraovarian cyst & 3 & - & - & 1 & - & 1 \\
\hline Endometrioma & 19 & 7 & 18 & 16 & 6 & 12 \\
\hline Serous cystadenoma & 5 & 2 & 1 & 1 & - & 5 \\
\hline Mucinous cystadenoma & 3 & 2 & - & - & - & 1 \\
\hline Dermoid cyst & 7 & 2 & 3 & 5 & - & 9 \\
\hline Cystic adenofibroma & 1 & - & - & 1 & - & - \\
\hline Corpus luteum cyst & 2 & - & 2 & 1 & - & - \\
\hline Borderline tumor & 1 & - & - & - & - & 1 \\
\hline Total & $50(47.6 \%)$ & $21(20.0 \%)$ & $25(23.8 \%)$ & $27(25.7 \%)$ & $7(6.7 \%)$ & $44(41.9 \%)$ \\
\hline
\end{tabular}

Table 3 Classification of ovarian tumors diagnosed in the patients of the study according to their age at presentation (in decades)

\begin{tabular}{|c|c|c|c|c|c|c|}
\hline & $<20$ & $21-30$ & $31-40$ & $41-50$ & $>50$ & Total \\
\hline Simple cyst & 2 & 13 & 12 & 3 & 2 & 32 \\
\hline Paraovarian cyst & 3 & - & 1 & - & - & 4 \\
\hline Endometrioma & 1 & 20 & 12 & 4 & - & 37 \\
\hline Serous cystadenoma & 2 & 3 & - & 2 & 1 & 8 \\
\hline Mucinous cystadenoma & - & - & 2 & 1 & 1 & 4 \\
\hline Dermoid cyst & 3 & 9 & 2 & 1 & - & 15 \\
\hline Cystic adenofibroma & - & - & 1 & - & - & 1 \\
\hline Corpus luteum cyst & - & 1 & 1 & - & - & 2 \\
\hline Borderline tumor & - & 2 & - & - & - & 2 \\
\hline Total & $11(10.5 \%)$ & $48(45.7 \%)$ & $31(29.5 \%)$ & $11(10.5 \%)$ & $4(3.8 \%)$ & $105(100 \%)$ \\
\hline
\end{tabular}

test with Yates correction were used to calculate any significant difference between numerical and nominal parameters respectively.

\section{Results}

Totally 98 women with 105 ovarian cysts were laparoscopically treated. There were 32 simple ovarian cysts $(30.48 \%), 4$ paraovarian cysts $(3.81 \%), 37$ endometriomas $(35.23 \%), 4$ mucinous cystadenomas $(3.81 \%), 8$ serous cystadenomas $(7.62 \%), 15$ dermoid cysts $(14.29 \%), 1$ cystadenofibroma $(0.95 \%), 2$ corpus luteum cysts $(1.9 \%)$, and 2 borderline ovarian tumors $(1.9 \%)$ (Table 1$)$. The main presenting symptoms were pelvic pain $(47.6 \%)$, dyspareunia $(25.7 \%)$, dysmenorrhoea (23.8\%), and menstrual disorders; $41.9 \%$ of the patients were asymptomatic presenting for their annual health visit (Table 2). Most of the patients diagnosed with ovarian tumors were 21-30 years old (45.7\%) and 31-40 years old (29.5\%); only four patients of this study (3.8\%) were older than 50 years (Table 3 ). The majority of simple cysts, endometriomas, and mucinous cystadenomas, had preoperative sonographic maximum diameter $3.1-5.0 \mathrm{~cm}$; the majority of paraovarian cysts, serous cystadenomas, and dermoid cysts, had preoperative sonographic maximal diameter $5.1-7.0 \mathrm{~cm}$ (Table 4). TVS identified correctly 30 cases of simple cysts (94\%), 3 paraovarian cysts (75\%), 29 endometriomas (78\%), 5 serous cystadenomas (63\%), 3 mucinous cystadenomas (75\%), and 12 dermoid cysts (80\%). Laparoscopically, were correctly diagnosed 30 cases of simple cysts $(94 \%), 4$ paraovarian cysts $(100 \%), 37$ endometriomas $(100 \%), 6$ serous cystadenomas $(75 \%), 3$ mucinous cystadenomas $(75 \%)$, and 15 dermoid cysts $(100 \%)$ (Table 5).

Regarding accuracy in the diagnosis of benign ovarian lesions, laparoscopy performed excellent in the detection of paraovarian cysts (sensitivity 100\%, specificity 100\%), endometriomas (sensitivity 100\%, specificity 100\%), dermoid cyst (sensitivity $100 \%$, specificity $100 \%$ ), very good in the detection of simple cysts (sensitivity $94 \%$, specificity 97\%), moderately good in the detection of serous (sensitivity $75 \%$, specificity 97\%), and mucinous cystadenomas (sensitivity $75 \%$, specificity $98 \%$ ). Ultrasound was proven very good in the diagnosis of simple (sensitivity $94 \%$, specificity 92\%) and dermoid cysts (sensitivity 80\%, specificity $92 \%$ ), moderately good in the detection of endometriomas 
Table 4 Histological findings in respect to the maximal sonographic cystic diameter in centimeter

\begin{tabular}{|c|c|c|c|c|c|c|}
\hline & $<3.0 \mathrm{~cm}$ & $3.1-5.0 \mathrm{~cm}$ & $5.1-7.0 \mathrm{~cm}$ & $7.1-9.0 \mathrm{~cm}$ & $>9.0 \mathrm{~cm}$ & Total \\
\hline Simple cyst & 2 & 14 & 13 & 2 & 1 & 32 \\
\hline Paraovarian cyst & - & 1 & 2 & - & 1 & 4 \\
\hline Endometrioma & 2 & 20 & 9 & 5 & 1 & 37 \\
\hline Serous cystadenoma & - & 2 & 4 & 2 & - & 8 \\
\hline Mucinous cystadenoma & - & 3 & 1 & - & - & 4 \\
\hline Dermoid cyst & 1 & 6 & 7 & 1 & - & 15 \\
\hline Cystic adenofibroma & - & 1 & - & - & - & 1 \\
\hline Corpus luteum cyst & - & - & 2 & - & - & 2 \\
\hline Borderline tumor & - & 1 & 1 & - & - & 2 \\
\hline Total & $5(4.7 \%)$ & $48(45.7 \%)$ & $39(37.1 \%)$ & $10(9.5 \%)$ & $3(2.9 \%)$ & $105(100 \%)$ \\
\hline
\end{tabular}

Table 5 Diagnostic accuracy of transvaginal ultrasound and laparoscopy in the diagnosis of benign adnexal masses

\begin{tabular}{|c|c|c|c|c|c|c|c|c|c|c|c|}
\hline & \multirow[t]{2}{*}{ Total } & \multicolumn{2}{|c|}{ Accurate diagnosis $(n)$} & \multicolumn{2}{|l|}{ Sensitivity } & \multicolumn{2}{|c|}{ Specificity } & \multicolumn{2}{|l|}{ PPV } & \multicolumn{2}{|l|}{ NPV } \\
\hline & & TVS & Lap & TVS (\%) & Lap (\%) & TVS $(\%)$ & Lap (\%) & TVS $(\%)$ & Lap (\%) & TVS $(\%)$ & Lap $(\%)$ \\
\hline Simple cyst & 32 & 30 & 30 & 94 & 94 & 92 & 97 & 83 & 94 & 97 & 97 \\
\hline Paraovarian cyst & 4 & 3 & 4 & 75 & 100 & 100 & 100 & 100 & 100 & 99 & 100 \\
\hline Endometrioma & 37 & 29 & 37 & 78 & $100^{\mathrm{a}}$ & 88 & $100^{\mathrm{a}}$ & 78 & 100 & 88 & 100 \\
\hline Serous cystadenoma & 8 & 5 & 6 & 63 & 75 & 97 & 97 & 63 & 67 & 97 & 98 \\
\hline Mucinous cystadenoma & 4 & 3 & 3 & 75 & 75 & 95 & 98 & 38 & 60 & 99 & 99 \\
\hline Dermoid cyst & 15 & 12 & 15 & 80 & 100 & 92 & 100 & 63 & 100 & 97 & 100 \\
\hline
\end{tabular}

$T V S$ transvaginal ultrasound, Lap laparoscopy, $P P V$ positive predictive value, $N P V$ negative predictive value

${ }^{a}$ Statistically significant difference

Table 6 Diagnostic accuracy of transvaginal ultrasound and laparoscopy in the detection of borderline tumors, benign tumors and endometriomas

\begin{tabular}{|c|c|c|c|c|c|c|}
\hline & \multicolumn{2}{|c|}{ Borderline tumors ${ }^{\mathrm{a}}$} & \multicolumn{2}{|c|}{ Benign tumors } & \multicolumn{2}{|c|}{ Endometriomas } \\
\hline & TVS (\%) & Lap (\%) & TVS (\%) & Lap $(\%)$ & TVS (\%) & Lap (\%) \\
\hline Sensitivity & 50 & 100 & 92 & 98 & 78 & $100^{\mathrm{b}}$ \\
\hline Specificity & 92 & 98 & 50 & 100 & 88 & $100^{\mathrm{b}}$ \\
\hline PPV & 11 & 50 & 99 & 100 & 78 & 100 \\
\hline NPV & 99 & 100 & 11 & 50 & 88 & 100 \\
\hline
\end{tabular}

TVS transvaginal ultrasound scan, Lap laparoscopy, $P P V$ positive predictive value, $N P V$ negative predictive value

a Sample size too small (two patients), not statistically significant

b Statistically significant difference

(sensitivity $78 \%$, specificity $88 \%$ ), paraovarian cysts (sensitivity $75 \%$, specificity $100 \%$ ), and mucinous cystadenomas (sensitivity $75 \%$, specificity $95 \%$ ), and good in the detection of serous cystadenomas (sensitivity $63 \%$, specificity 97\%) (Table 5).

Regarding accuracy in the diagnosis of borderline tumors, laparoscopy had sensitivity of $100 \%$ and specificity of $98 \%$. Ultrasound examination had lower sensitivity $(50 \%)$ and specificity $(92 \%)$ in the detection of ovarian borderline tumors.
Although laparoscopy showed an overall higher performance compared to transvaginal ultrasound, statistically significant difference was found only in the detection of endometriomas compared to ultrasound ( $P=0.004$ for sensitivity and $P=0.046$ for specificity) (Table 6 ).

Mean hospitalization was $0.98 \pm 0.95$ days (0-3 days). Oophorectomy was performed in eight cases: a case with a borderline tumor, two cases with mucinous cystadenomas, two cases with serous cystadenomas, and three cases with simple ovarian cysts. Conversion to laparotomy was 
decided in two cases (2/98): in the first case endometriosis stage IV with bilateral endometriomas and extended pelvic adhesions (frozen pelvis) was intraoperatively encountered, and decision for laparotomy was decided upon the high risk of bowel damage that the laparoscopic approach involved; in the second case there were preoperative indications of highly suspicious for malignancy ovarian tumor (TVS showed intracystic vegetations and septa), and during laparoscopy external cystic vegetations were recognized (histology confirmed borderline ovarian tumor). There were four intra- and postoperative complications: a case of subcutaneous emphysema, one case of postoperative shoulder pain, one case of large bowel serosa laceration [the lesion was detected and sutured with $3 / 0$ polyglycolic acid suture (Vicryl, Ethicon) laparoscopically], one case of urinary tract infection.

The age of women with borderline tumors was not statistically different compared to the age of women with any other ovarian lesion. Dermoid cysts presented in women of younger age compared to simple cysts $(P=0.024)$, endometriomas $(P=0.003)$, and mucinous cystadenomas $(P=0.000)$. Mucinous cystadenomas presented in elder women compared to endometriomas $(P=0.009)$.

Seven women presented with bilateral ovarian tumors. Bilateral endometriomas were found in three of them, bilateral simple ovarian cysts in one, and combination of endometrioma with simple cyst, mucinous cystadenoma with simple cyst and borderline tumor with simple cyst in three different subjects.

\section{Discussion}

Despite the recent advances in the imaging technology, the current clinical use of transvaginal sonography with or without the aid of color Doppler, combined with the serum markers is not always adequate to distinguish between benign, borderline, and malignant ovarian tumors [12]. The occurrence of borderline (low malignant potential) ovarian masses is $10-20 \%$ of all ovarian epithelial tumors and is mainly diagnosed in young women [13]. More importantly, it has been described that in preoperatively selected patients with adnexal cystic masses without sonographic evidence of thick septa, internal wall papillae, or solid components, the rate of laparoscopically discovered adnexal cysts with intracystic papillary projections was $5 \%$ of which $14 \%$ were borderline tumors [14].

In our series, the diagnostic accuracy of laparoscopy in the identification of ovarian lesions was higher compared to ultrasound examination. In the diagnosis of borderline ovarian tumors laparoscopy had excellent sensitivity and specificity (100 and 98\% respectively), with positive predictive value $50 \%$ and negative predictive value $100 \%$. Laparoscopy set the diagnosis correctly in all the cases of endometriomas, dermoid cysts, and paraovarian cysts in our series. Laparoscopy was less accurate in the description of the nature of serous and mucinous cystadenomas, although the lower sensitivity laparoscopy exhibits in these cases is mostly unimportant, because it does not alter the conservative surgical approach that consists of ovarian cystectomy in such cases.

Generally, our results suggest that sensitivity and specificity of the laparoscopic diagnosis of benign and borderline ovarian lesions are rather superior compared to the ultrasound approach (Table 6). Laparoscopic diagnosis of endometriomas was statistically more accurate compared to the sonographic examination of these lesions $(P=0.004$ for the sensitivity and $P=0.046$ for the specificity in the comparison of these methods). Due to the small sample size (one case of cystic adenoma, two cases of corpus luteum cysts, four cases of paraovarian cysts, and four cases of serous cystadenomas), there is no statistical difference in the diagnostic performance of laparoscopy compared to ultrasound in certain types of benign ovarian lesions. There is also no statistical difference in laparoscopic compared to the sonographic evaluation in simple ovarian cysts (both methods appear to have $94 \%$ sensitivity in the diagnosis of simple cysts).

As our results suggest, the feasibility of laparoscopic diagnosis is apparent in the management of benign ovarian tumors. This is in agreement with modern literature, as even laparoscopic management of borderline ovarian tumors is feasible and safe when oncological surgical principles are respected $[15,16]$. In a series of 819 women treated laparoscopically for ovarian tumors, laparoscopy had $100 \%$ sensitivity in the detection of malignant lesions compared to histology, with negative and positive predictive values 41.3 and $100 \%$ respectively [3]. Guidelines for surgical management of these lesions are similar to those of ovarian malignancy and include laparotomy with peritoneal washing, hysterectomy and bilateral salpingoophorectomy, and multiple peritoneal biopsies [17]. The laparoscopic staging in women treated for borderline ovarian tumors is comparable to the laparotomic one [18]. Independently of the high suspicion an adnexal mass may appear in the sonographic investigation, an attempt for laparoscopic diagnosis is valuable for a number of reasons: (a) in cases of non-neoplastic but post-inflammatory lesions, laparotomy is avoided with certain benefits for the patient, (b) in cases of diagnosis of ovarian cancer, initial minimally invasive approach allows midline laparotomy for as per protocol management of the lesions, and (c) in cases of ovarian cancer laparoscopic magnification provides the chance to detect microscopic metastatic lesion in the peritoneum, especially in upper abdomen and 
subdiaphragmatically [19]. On the other hand, drawbacks of laparoscopic management of adnexal masses include: (a) the possible non-detection of a malignant lesion or the possibility of not converting to laparotomy, especially in cases where operation takes place in day-surgery units, or by inexperienced surgeons, (b) the rupture of the ovarian cyst and the intraperitoneal spillage of the contents (possibly malignant) of the tumor, and (c) the port-site metastasis and the intraperitoneal transfer of the malignant cells $[16,19]$.

In our series, TVS's diagnostic performance in the investigation of borderline ovarian tumors was quite satisfactory (sensitivity $50 \%$, specificity $92 \%$, positive predictive value $11 \%$, and negative predictive value 99\%). Compared to laparoscopic diagnosis, however, the performance of TVS is slightly poorer, but not statistically significant different, probably due to the small sample size $(n=2)$. As mentioned above, endometriomas were less accurately detected with ultrasound. In the sonographic investigation of the rest of the benign ovarian lesions studied (simple cysts, cystadenomas, dermoid cysts, paraovarian cysts), no statistical difference was found compared to laparoscopy.

The use of color Doppler in the investigation of ovarian tumors has been extensively studied, and specific predictive for malignancy values for pulsatility index $(\mathrm{PI}<1.0)$ and resistance index $(\mathrm{RI}<0.4)$ have been proposed [20]. Nevertheless, PI and RI cut off values for benign, borderline, and malignant ovarian tumors described by various researchers are in the same range [21]. This lack of sensitivity makes color Doppler a technique of limited value in the investigation of ovarian lesions [22]. Main reason of the poor distinctive ability of color Doppler is that in cases of ovarian malignancies there is non specific neovascularization. In accordance to this, in a prospective study where clinical examination, TVS, serum markers, and color Doppler, were evaluated in the preoperative investigation of ovarian tumors, the size of the lesion and the TVS morphologic features were the best predictive markers for malignancy in the premenopausal women, and the morphology and CA-125 for the postmenopausal women [22]. The role of other advanced imaging modalities like magnetic resonance imaging (MRI), computed tomography (CT), and positron emission tomography (PET) in the diagnosis of borderline or malignant ovarian tumors is still under debate. Contrast enhanced MRI appears to have $85-95 \%$ sensitivity and $87-96 \%$ specificity in the diagnosis of ovarian malignancies [23]. Nevertheless, according to Stratton et al., MRI has lower diagnostic accuracy compared to laparoscopy in the detection of endometriomas [24]. CT and PET do not appear to offer more information compared to the ultrasound scan [25].

\section{Conclusion}

In conclusion, this study demonstrates the feasibility of laparoscopic diagnosis in the investigation of benign and borderline ovarian tumors and the higher diagnostic accuracy, especially in endometriomas, of laparoscopy, compared to transvaginal scan, especially in endometriomas. Laparoscopic diagnosis appears to be safe and accurate. Conservative laparoscopic management of benign adnexal masses is safe and with low morbidity.

\section{Conflict of interest statement None}

Open Access This article is distributed under the terms of the Creative Commons Attribution Noncommercial License which permits any noncommercial use, distribution, and reproduction in any medium, provided the original author(s) and source are credited.

\section{References}

1. Parker WH, Berek J (1994) Laparoscopic management of the adnexal mass. Obstet Gynecol Clin North Am 21:79-92

2. Nezhat F, Nezhat C, Welander CE et al (1992) Four ovarian cancers diagnosed during laparoscopic management of 1011 women with adnexal masses. Am J Obstet Gynecol 167:790-796

3. Canis M, Mage G, Pouly JL et al (1994) Laparoscopic diagnosis of adnexal cystic masses: a 12-year experience with long-term follow-up. Obstet Gynecol 83:707-712

4. Mettler L, Schollmeyer T, Lehmann-Willenbrock E et al (2003) Accuracy of laparoscopic diagnosis of endometriosis. JSLS 7:15-18

5. Marana R, Muzii L, Catalano GF et al (2004) Laparoscopic excision of adnexal masses. J Am Assoc Gynecol Laparosc 11:162166. doi:10.1016/S1074-3804(05)60191-0

6. Sassone AM, Timor-Tritch IE, Artner A et al (1991) Transvaginal sonographic characterization of ovarian disease: evaluation of a new scoring system to predict ovarian malignancy. Obstet Gynecol 78:70-76

7. DePriest PD, Shenson D, Fried A et al (1993) A morphology index based on sonographic findings in ovarian cancer. Gynecol Oncol 51:7-11. doi:10.1006/gyno.1993.1238

8. Bromley B, Goodman H, Benacerraf BR (1994) Comparison between sonographic morphology and Doppler waveform for the diagnosis of ovarian malignancy. Obstet Gynecol 83:434-437

9. Kinkel K, Hricak H, Lu Y et al (2000) US characteristics of ovarian masses: a meta-analysis. Radiology 217:803-811

10. Maruiri F, Azziz A (1993) Laparoscopic surgery for ectopic pregnancies: technology assessment and public health implications. Fertil Steril 59:487-498

11. Mais V, Ajossa S, Guerriero S et al (1996) Laparoscopic versus abdominal myomectomy: a prospective randomized trial to evaluate benefits in early outcome. Am J Obstet Gynecol 174:654-658. doi:10.1016/S0002-9378(96)70445-3

12. Fauvet R, Boccara J, Dufournet C et al (2005) Laparoscopic management of borderline ovarian tumors: results of a French multicenter study. Ann Oncol 16:403-410. doi:10.1093/annonc/mdi083

13. Hoskins PJ (1995) Ovarian tumors of low malignant potential: borderline epithelial ovarian carcinoma. In: Lawton FG, Neijt JP, Swenerton KD (eds) Epithelial cancer of the ovary. BMJ Publishing Group, London, pp 112-136

14. Marana R, Muzii L, Ferrari S et al (2005) Management of adnexal cystic masses with unexpected intracystic vegetations detected 
during laparoscopy. J Minim Invasive Gynecol 12:502-507. doi:10.1016/j.jmig.2005.09.003

15. Seracchioli R, Venturoli S, Colombo FM et al (2001) Fertility and tumor recurrence rate after conservative laparoscopic management of young women with early-stage borderline ovarian tumors. Fertil Steril 76:999-1004. doi:10.1016/S0015-0282(01)02842-4

16. Theodoridis TD, Bontis J (2003) Laparoscopy and oncology: where do we stand today? Ann NY Acad Sci 997:282-291. doi:10.1196/annals.1290.032

17. International Federation of Gynaecology, Obstetrics (1989) Annual report and results of treatment in gynaecologic cancer. Int J Gynaecol Obstet 28:189-190. doi:10.1016/0020-7292(89)90482-7

18. Querleu D, Papageorgiou T, Lambaudie E et al (2003) Laparoscopic restaging of borderline ovarian tumours: results of 30 cases initially presumed as stage IA borderline ovarian tumours. $\mathrm{Br}$ J Obstet Gynaecol 110:201-204

19. Childers JM, Lang J, Surwit EA et al (1995) Laparoscopic surgical staging of ovarian cancer. Gynecol Oncol 59:25-33. doi:10.1006/ gyno. 1995.1263

20. Kurjak A, Predanic M (1992) New scoring system for prediction of ovarian malignancy based on transvaginal color Doppler sonography. J Ultrasound Med 11:631-638
21. Kurjak A, Kupesic S, Simunic V (2002) Ultrasonic of the peri- and postmenopausal ovary. Maturitas 41:245-254. doi:10.1016/ S0378-5122(01)00305-X

22. Roman LD, Muderspach LI, Stein SM et al (1997) Pelvic examination, tumor marker level, and gray scale and Doppler sonography in the prediction of pelvic cancer. Obstet Gynecol 89:493500. doi:10.1016/S0029-7844(97)00075-6

23. Rieber A, Nossle K, Stiehr I et al (2001) Preoperative diagnosis of ovarian tumors with MR imaging comparison with transvaginal sonography, positron emission tomography, and histologic findings. AJR Am J Roentgenol 177:123-129

24. Stratton P, Winkel C, Premkumar A et al (2003) Diagnostic accuracy of laparoscopy, magnetic resonance imaging, and histopathologic examination for the detection of endometriosis. Fertil Steril 79:1078-1085. doi:10.1016/S0015-0282(03)00155-9

25. Walsh JW, Taylor KJW, Wasson JFM (1978) Prospective comparison of ultrasound and computed tomography in the evaluation of gynecologic pelvic masses. AJR Am J Roentgenol 131:955-959 